\title{
Successful removal of a peripherally inserted central catheter fragment from the heart with a vena cava filter retrieval set
}

\author{
Nan-Dong Miao, Hao Xu, Lin Yang*, Xiao-Ming Zhang, Yong-Jun Ren, Kang Liu and Xu-Li Min \\ *Correspondence: linyangmd@163.com

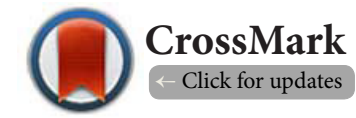 \\ Sichuan Key Laboratory of Medical Imaging, Department of Radiology, Affiliated Hospital of North Sichuan Medical College, \\ Nanchong, Sichuan 637000, P. R. China.
}

\begin{abstract}
Many major complications related to peripherally inserted central catheter (PICC) lines have been reported in the literature. Of these complications, spontaneous catheter fracture is rare. Percutaneous retrieval avoids the need for surgery in these cases. A few percutaneous techniques have been reported, but very little has been published in the literature regarding the use of vena cava filter retrieval sets. Here, we report a case of a female patient with breast cancer who experienced a fracture of the PICC with subsequent migration of the catheter fragment through the superior vena cava and into the right atrium, the right ventricle and the main pulmonary artery. The catheter fragment was removed by retrograde femoral vein catheterization with a vena cava filter retrieval set.
\end{abstract}

Keywords: Radiology, interventional, complications, foreign bodies

\section{Introduction}

Peripherally inserted central catheters (PICC) are widely used to provide central venous access in chronically ill patients with long-term intravenous access requirements. Many major complications related to both the insertion and the maintenance of PICC lines have been reported in the literature [1]. Typical complications include pneumothorax, hemopneumothorax, malposition, migration, catheter-associated infection and venous thrombosis. Of these complications, spontaneous catheter fracture is rare. Percutaneous retrieval avoids the need for surgery in these cases. A few percutaneous techniques have been reported, but very little has been published in the literature regarding the use of vena cava filter retrieval sets $[2,3]$. Here, we report a case of a female patient with breast cancer who experienced a fracture of the PICC with subsequent migration of the catheter fragment through the superior vena cava and into the right atrium, the right ventricle and the main pulmonary artery. The catheter fragment was removed by retrograde femoral vein catheterization with a vena cava filter retrieval set.

\section{Patient data}

A 43-year-old woman was hospitalized for chemotherapy for cancer of the right breast. A PICC was placed through the left cubital vein to the superior vena cava for this purpose (Figure 1). The PICC was removed two months after the completion of chemotherapy, but it was not found to be intact. Emergency chest radiography confirmed a catheter fragment in the area of the right atrium, the right ventricle and the main pulmonary artery (Figure 2). In view of the patient's risk for embolism, it was decided to remove the embolized catheter fragment. Using a percutaneous right femoral venous approach, the $11 \mathrm{~F}$ coaxial retrieval sheath system of a vena cava filter retrieval set (WILLIAM COOK EUROPE, Sandet 6, DK-4632, Bjaeverskov, Denmark) was advanced to the right atrium over the wire guide; then, the inner coaxial catheter and the wire guide were removed. The position of the coaxial retrieval sheath system was verified by the injection of contrast medium. The retrieval loop system was introduced through the coaxial retrieval sheath system, and the PICC fragment was removed intact and without complications. A follow-up chest radiograph excluded the presence of any residual fragments in the superior vena cava and the right atrium (Figure 3). The patient was discharged in stable condition on the 4th day after the transcatheter procedure.

\section{Discussion}

A broken catheter can migrate distally through the bloodstream to finally lodge in the vena cava, the right atrium, the right 


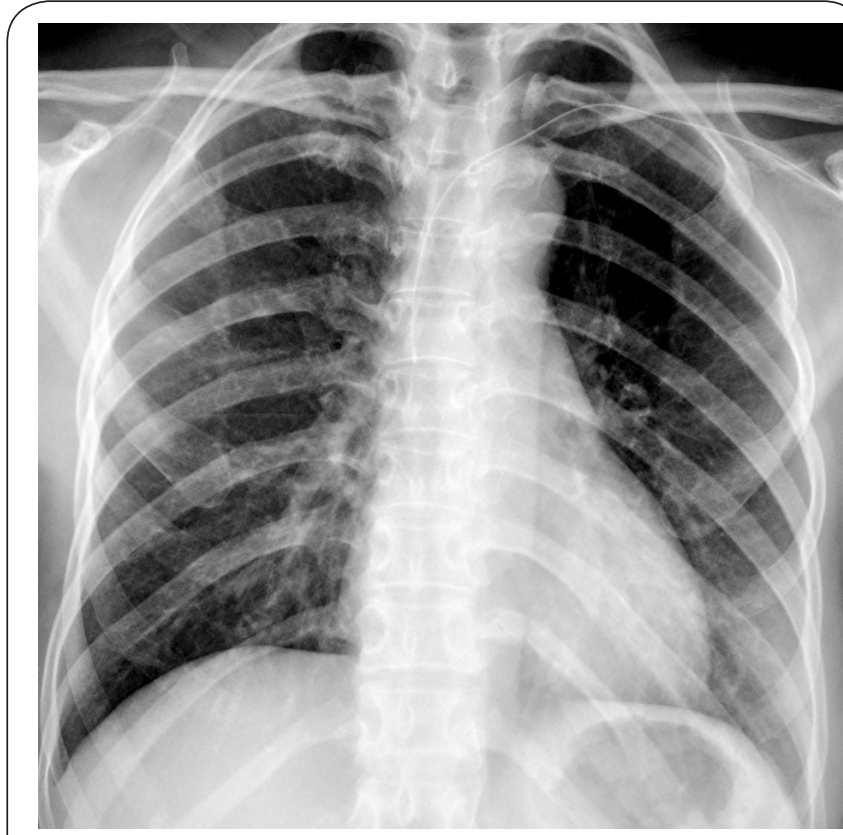

Figure 1. Chest radiograph showing the peripherally inserted central catheter placed through the left cubital vein to the superior vena cava.

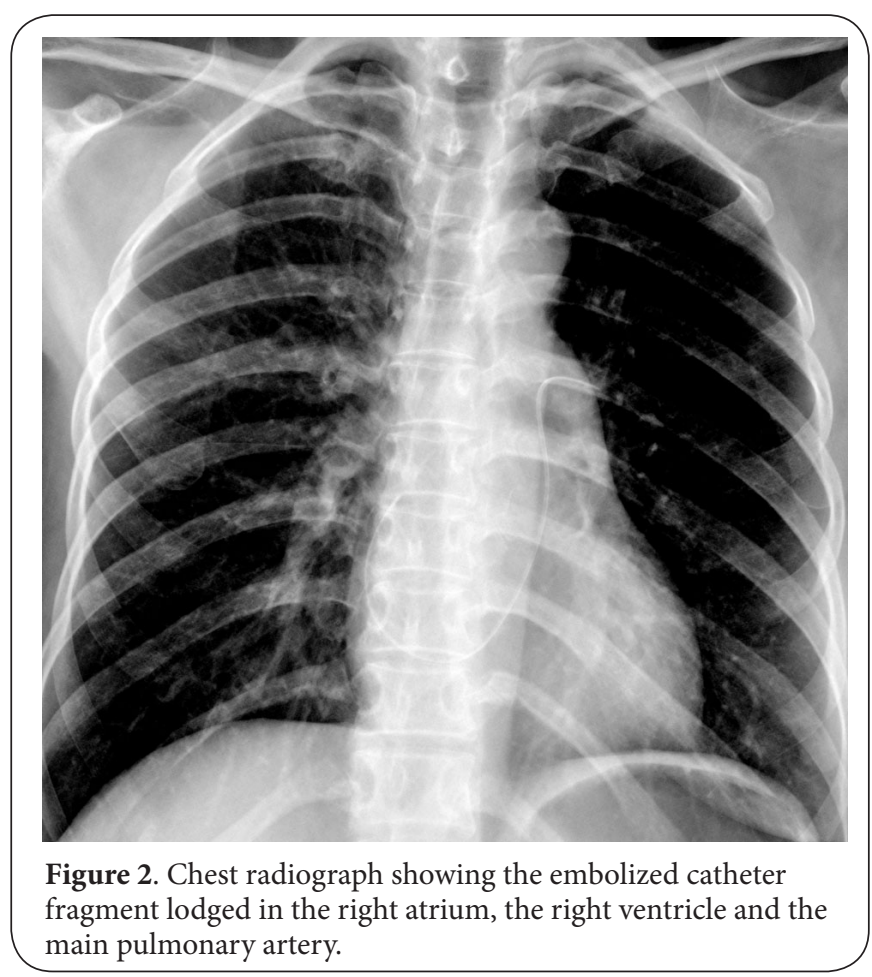

ventricle, or the main pulmonary/artery or one of its branches. In this case, the catheter fragment was finally located in the right atrium, the right ventricle and the main pulmonary artery.

It has been reported that centrally embolized foreign bodies may be associated with serious complications, including

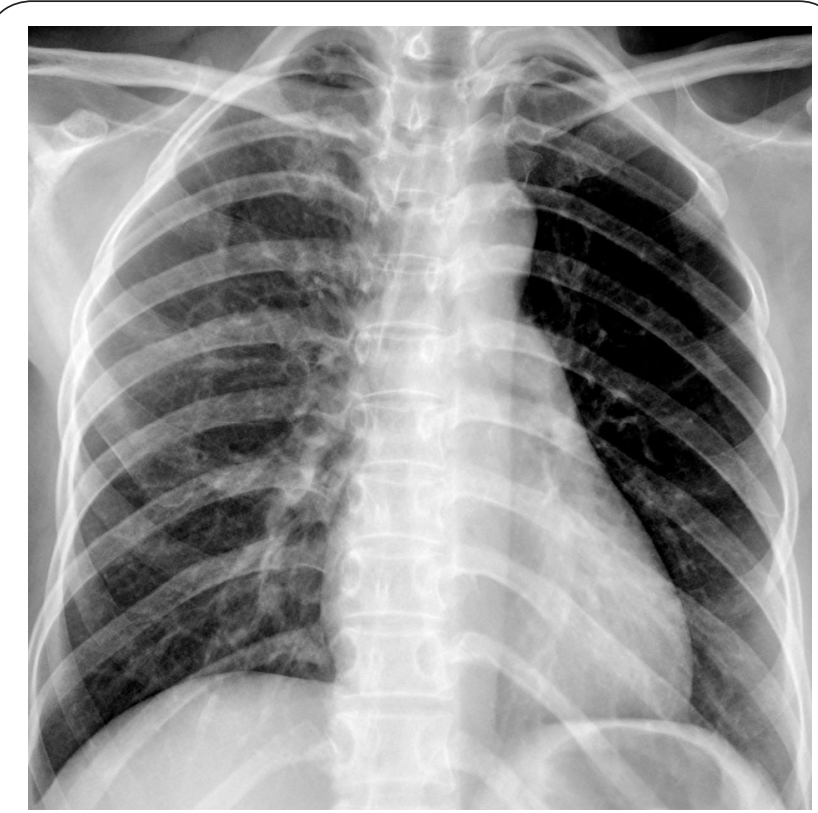

Figure 3. Chest radiograph after percutaneous retrieval of the embolized catheter fragment, demonstrating the absence of any residual fragments.

myocardial or valvular perforation, myocardial infarction, arrhythmia, cardiac arrest, endocarditis, pulmonary embolism and secondary infection of thrombus or pulmonary abscesses [4]. Although some patients may be completely asymptomatic or may suffer no complications from the retained catheter fragment for many years [5], the risk of such complications is considerable [6]. In view of these risks, an attempt should be made at removal [4]. If percutaneous retrieval fails, surgical retrieval may be necessary. Percutaneous retrieval can be performed using loop snares, Dormia baskets, hooked guide wires, and Fogarty balloon catheters. In this case, we successfully used a vena cava filter retrieval set to remove the catheter fragment.

\section{Conclusion}

Percutaneous retrieval is the preferred method for the removal of intracardiac catheter fragments. Our experience demonstrates that vena cava filter retrieval sets are useful equipment for the removal of PICC fragments from the heart.

\section{Competing interests}

The authors declare that they have no competing interests.

\section{Authors' contributions}

\begin{tabular}{|l|c|c|c|c|c|c|c|}
\hline Authors' contributions & NDM & HX & LY & XMZ & YJR & KL & XLM \\
\hline Research concept and design & $\checkmark$ & $\checkmark$ & $\checkmark$ & $\checkmark$ & $\checkmark$ & $\checkmark$ & $\checkmark$ \\
\hline Collection and/or assembly of data & $\checkmark$ & $\checkmark$ & $\checkmark$ & -- & -- & -- & -- \\
\hline Data analysis and interpretation & $\checkmark$ & $\checkmark$ & $\checkmark$ & $\checkmark$ & $\checkmark$ & $\checkmark$ & $\checkmark$ \\
\hline Writing the article & $\checkmark$ & $\checkmark$ & $\checkmark$ & -- & -- & -- & -- \\
\hline Critical revision of the article & -- & -- & $\checkmark$ & $\checkmark$ & -- & -- & -- \\
\hline Final approval of article & $\checkmark$ & $\checkmark$ & $\checkmark$ & $\checkmark$ & $\checkmark$ & $\checkmark$ & $\checkmark$ \\
\hline
\end{tabular}


Miao et al. Cardiovascular System 2014,

\section{Acknowledgement}

The authors wish to thank Yong Luo in the Department of Cardiology, and Yanchun Gao in the Department of General surgery, Affiliated Hospital of North Sichuan Medical College, for their assistance in all aspects of patient care and management for this study.

\section{Publication history}

Senior Editor: Shiwei Duan, Ningbo University, China.

EIC:William Clifford Roberts, Baylor University Medical Center, USA. Received: 21-Apr-2014 Final Revised: 02-Jul-2014

Accepted: 23-Jul-2014 Published: 28-Jul-2014

\section{References}

1. Amerasekera SS, Jones CM, Patel R and Cleasby MJ. Imaging of the complications of peripherally inserted central venous catheters. Clin Radiol. 2009; 64:832-40. | Article | PubMed

2. Haase R, Lieser U, Nagel F and Syska J. [Removal of a broken silastic catheter with a "snare"-catheter in a neonate]. Z Geburtshilfe Neonatol. 2007; 211:33-5. | Article | PubMed

3. Thanigaraj S, Panneerselvam A and Yanos J. Retrieval of an IV catheter fragment from the pulmonary artery 11 years after embolization. Chest. 2000; 117:1209-11. | Article | PubMed

4. Andrews RE, Tulloh RM and Rigby ML. Percutaneous retrieval of central venous catheter fragments. Arch Dis Child. 2002; 87:149-50. | Article | PubMed Abstract | PubMed Full Text

5. Thanigaraj S, Panneerselvam A and Yanos J. Retrieval of an IV catheter fragment from the pulmonary artery 11 years after embolization. Chest. 2000; 117:1209-11. | Article | PubMed

6. Roye GD, Breazeale EE, Byrnes JP and Rue LW, 3rd. Management of catheter emboli. South Med J. 1996; 89:714-7. | PubMed

\section{Citation:}

Miao N-D, Xu H, Yang L, Zhang X-M, Ren Y-J, Liu K and Min X-L. Successful removal of a peripherally inserted central catheter fragment from the heart with a vena cava filter retrieval set. Cardio Vasc Syst. 2014; 2:7. http://dx.doi.org/10.7243/2052-4358-2-7 